SHORT REPORT

\title{
The value of an elastic tissue stain in detecting venous invasion in colorectal cancer
}

\author{
D G Vass, R Ainsworth, J H Anderson, D Murray, A K Foulis
}

J Clin Pathol 2004;57:769-772. doi: 10.1136/icp.2003.015826

Background: Venous invasion by tumour is an independent prognostic indicator of both prognosis and risk of development of distant metastases in colorectal carcinoma. The use of special stains to aid its detection in pathology specimens is not currently universally recommended.

Aims: To determine whether an elastica stain significantly increases the incidence of detection of vascular invasion compared with routinely stained sections.

Methods: Serial sections from the 75 cases of colorectal carcinoma were stained by haematoxylin and eosin (H\&E) only and elastica counterstained with H\&E. The incidence of both intramural and extramural venous invasion was recorded and compared with that seen when the tumours were originally reported.

Results: Extramural venous invasion had been noted in 14 of the pathology reports and was seen in 18 cases when only the H\&E sections were viewed in the study. It was present in 32 cases when elastica stained sections were analysed. Intramural venous invasion was seen in eight cases on H\&E sections and 30 cases on elastica stained sections.

Conclusion: The use of elastica stained serial sections to detect venous invasion in tumours should be recommended in guidelines for the reporting of colorectal carcinomas.

$\mathrm{V}$ enous invasion is an important prognostic indicator in colorectal cancer. It is well established that blood vessel invasion, found in the tumour at the time of resection, is associated with a significantly increased risk of visceral metastases and a decrease in overall survival time. This observation is independent of Dukes's stage and degree of differentiation. ${ }^{1-6}$

Pathological assessment of venous invasion was largely ignored until Brown and Warren highlighted its prognostic implications in 1938. ${ }^{1}$ In a postmortem review they examined 170 patients who had died of rectal cancer. Using macroscopic dissection, venous invasion was detected in $61 \%$ of cases, and of this cohort $71 \%$ had synchronous liver metastases.

The prevalence of venous invasion in previous studies has ranged between $10 \%$ and $90 \% .{ }^{1-6}$ This wide range may result from selection bias of advanced cases in some series, ${ }^{6}$ or variations in the methods of pathological analysis performed. Elastic fibres are present in the adventitia of veins (but not lymphatics) and elastica stains can be used to highlight the presence of veins and their adjacent arteries. The use of elastic tissue stains in microscopic assessment has been proposed as being a more sensitive means of revealing venous invasion within the tumour, but has failed to gain widespread acceptance-for example, this technique is not recommended in the pathology guidelines for reporting colorectal cancers, produced by the Royal College of Pathologists. $^{7}$
The aims of our present retrospective study of colorectal cancer resections were:

(1) To undertake an audit of the original general pathologists' ability to detect venous invasion in a routine reporting setting, by comparing the incidence of venous invasion reported in the original pathology report with that found in our study on freshly cut haematoxylin and eosin (H\&E) stained sections of each tumour.

(2) To assess the sensitivity of detection of venous invasion using an elastica special stain, which highlights veins, and compare this with the incidence found in serial H\&E sections.

\section{METHODS}

Thirty nine randomly selected cases of colon cancer and 36 randomly selected cases of rectal cancer surgically resected at the Glasgow Royal Infirmary, UK between the years 1997 and 2000 were examined retrospectively. Curative and palliative resections were included in the cohort. In all cases included in our study there was sufficient tumour material for assessment (at least two tumour blocks) and the patient had not received preoperative radiotherapy or chemotherapy.

All cases had been reported at the time of receipt using a proforma, based on the minimum data set drafted by the Royal College of Pathologists, which specifically asked whether extramural vascular invasion (invasion of veins external to the muscularis propria of the bowel wall) was present or not. ${ }^{7}$

Blocks of tissue had been fixed in 10\% buffered formalin, processed in the usual manner, and embedded in paraffin wax. After retrieval from the archives, two serial sections of $4 \mu \mathrm{m}$ thickness were cut from each tumour containing block. One section was stained with H\&E only; the other was stained with Miller's elastic stain (BDH, Poole, Dorset, UK) and counterstained with H\&E.

Histological material was analysed independently by three of the authors (AKF, a consultant pathologist with a special interest in gastrointestinal pathology; RA, a trainee pathologist; and DGV, a medical student), blinded as to how the case had been reported originally. First, to simulate the method used in the department at the time the cases were originally examined, the $H \& E$ sections were examined with no reference to the elastica stained sections. Second, to simulate the reporting situation if an elastica stain were to be used routinely, cases were examined by viewing both the H\&E and H\&E/elastica stained sections. These two exercises were not done on the same day.

Venous invasion in H\&E sections was defined as a tumour deposit in a space surrounded by a rim of smooth muscle and/or containing red blood corpuscles, usually lying adjacent

Abbreviations: EVG, Verhoeff van Gieson elastic; H\&E, haematoxylin and eosin 


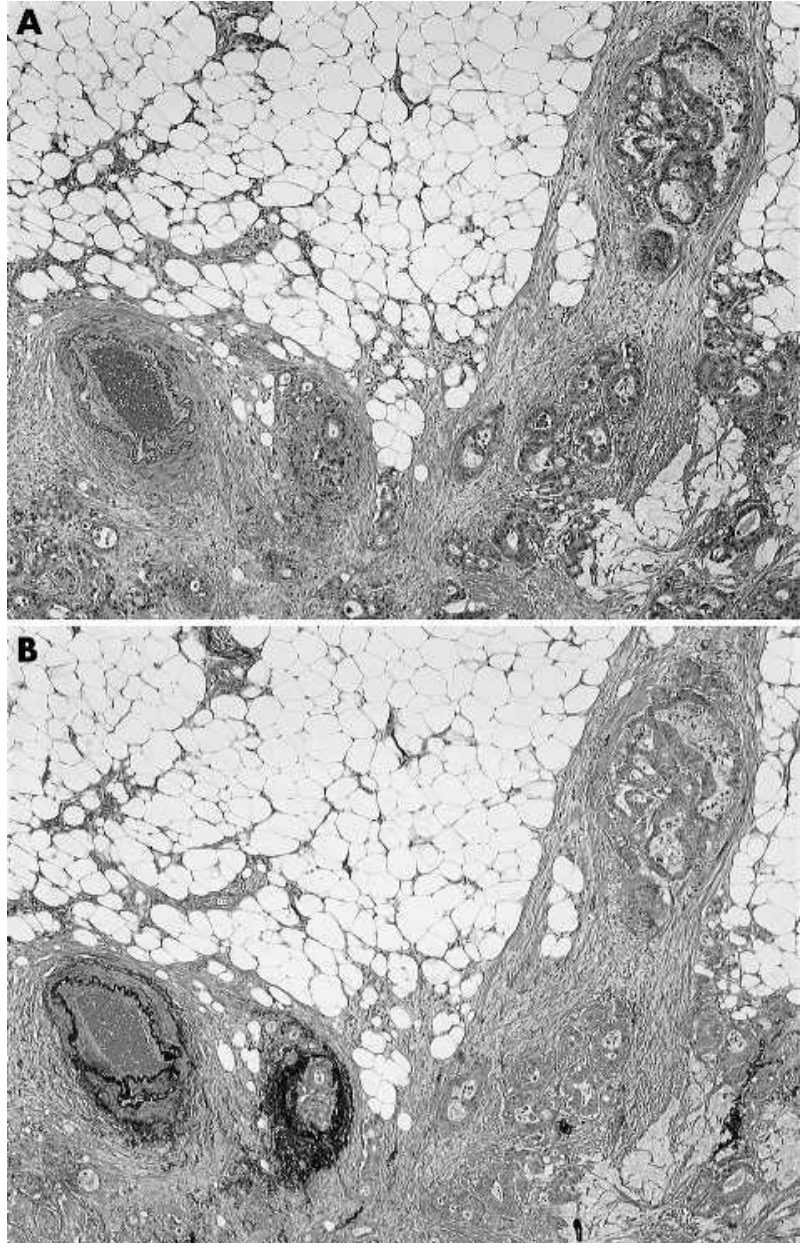

Figure 1 Extramural venous invasion: in this section of the leading edge of the tumour an artery is seen at the bottom left of the picture. (A) Haematoxylin and eosin (H\&E) staining: venous invasion is difficult to detect. (B) H\&E and elastica staining: a small extramural vein, to the right of the artery, is seen to contain tumour.

to an artery. Venous invasion was further defined in the H\&E/elastica sections as tumour seen in a vessel with elastic fibres in its adventitia, usually adjacent to an artery. Sections were assessed for the presence of both extramural and intramural venous invasion (invasion of veins in the submucosa and within the muscularis propria). If it was uncertain whether a structure was venous in origin the case was reported as being negative for invasion of this structure.

Where there was a disagreement between the participants the slides were re-examined and sections marked to show what was considered venous invasion. If consensus was still not reached a discussion at a double headed microscope resolved the issue.

The results using the two stains were compared statistically using the $\chi^{2}$ test

\section{RESULTS}

Extramural venous invasion was recorded in 14 of the original reports. In our present study, examination of fresh H\&E stains revealed this phenomenon in 18 (no significant difference). In four cases, extramural venous invasion was seen only on the original slides and in eight cases only on the new H\&E slides. In the other 10 cases this form of vascular invasion was seen in both. In contrast, examination of the H\&E elastica stain showed extramural venous invasion in 32 ( $\mathrm{p}<0.001$ compared with new H\&E sections; table 1 ; fig 1 ).
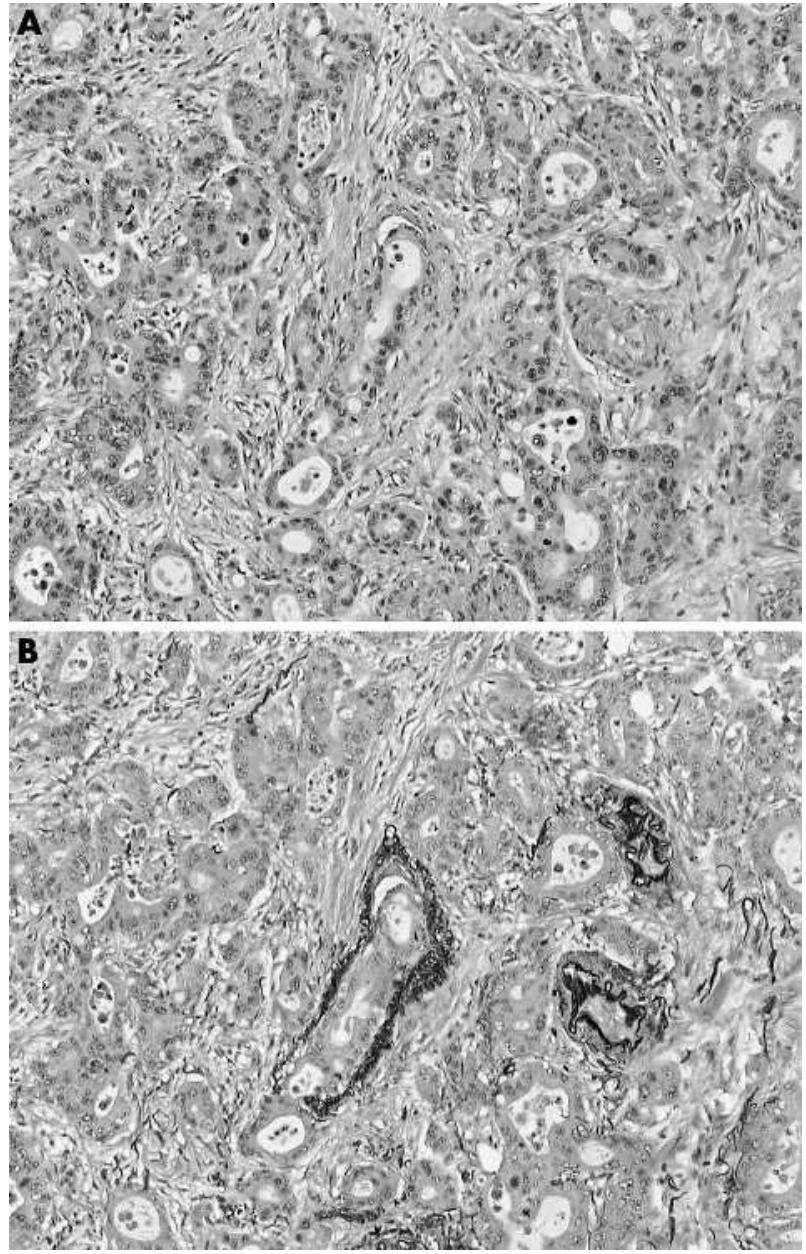

Figure 2 Intramural venous invasion: (A) even at high magnification venous invasion is difficult to see on the haematoxylin and eosin stained section. (B) The elastica stained section shows a vein (centre) invaded by tumour. Two cross sections of a small artery are seen to the right of this.

Intramural venous invasion was not routinely looked for in the original reports because this finding is not required for the minimum data set of the Royal College of Pathologists. In our present study it was seen in eight cases in the H\&E stained sections but in 30 cases stained by H\&E elastica $(\mathrm{p}<0.001$; table 1; fig 2$)$.

In all cases where either intramural or extramural venous invasion was seen on $\mathrm{H} \& \mathrm{E}$ it was also seen on $\mathrm{H} \& \mathrm{E} /$ elastica stained sections-that is, there was no false positive reporting on H\&E sections.

There was no obvious difference in the conclusions when analysing colonic and rectal carcinoma cases separately (table 1). Results were also analysed in relation to Dukes's staging (table 2). Finally, the number of sections examined for each case was analysed in relation to the finding of venous invasion of any type in elastica stained sections (table 3).

\section{DISCUSSION}

At the time when these cases were being reported in the department (1997-9), there was no attempt to arrange specialist reporting of colorectal carcinoma specimens and they were reported by most of the consultants and senior medical trainees on the staff. However, all were reported using a proforma based on the Royal College of Pathologists' minimum data set, which specifically required that the presence or absence of extramural venous invasion should be 
Table $1 \mathrm{VI}$ in relation to site of origin

\begin{tabular}{|c|c|c|c|c|c|c|c|c|}
\hline \multirow[b]{3}{*}{ Site } & \multirow[b]{3}{*}{$\mathrm{N}$} & \multicolumn{7}{|l|}{ Study results } \\
\hline & & \multicolumn{2}{|l|}{ Intramural VI } & \multicolumn{2}{|c|}{ Extramural VI } & \multicolumn{2}{|l|}{ Overall VI } & \multirow{2}{*}{$\begin{array}{l}\text { Extramural } \mathrm{VI} \text { in } \\
\text { original report }\end{array}$} \\
\hline & & H\&E (\%) & Elastica (\%) & H\&E (\%) & Elastica (\%) & H\&E (\%) & Elastica (\%) & \\
\hline Colon & 39 & $3(8)$ & $14(36)^{\star * *}$ & $6(15)$ & $14(36)^{\star \star}$ & $8(21)$ & $21(54)^{\star \star \star}$ & $6(15)$ \\
\hline Rectum & 36 & $5(14)$ & $16(44)^{* * *}$ & $12(33)$ & $18(50)^{*}$ & $13(36)$ & $22(61)^{* *}$ & $8(22)$ \\
\hline Total & 75 & $8(11)$ & $30(40)^{* * *}$ & $18(24)$ & $32(43)^{\star \star * *}$ & 21 (27) & $43(57)^{\star \star *}$ & 14 (19) \\
\hline
\end{tabular}

${ }^{*} \mathrm{p}<0.05 ;{ }^{* *} \mathrm{p}<0.01 ;{ }^{* * *} \mathrm{p}<0.001$ by $\chi^{2}$ test.

$\mathrm{H} \& \mathrm{E}$, haematoxylin and eosin; VI, venous invasion.

recorded. ${ }^{7}$ The incidence of extramural venous invasion documented in the pathology reports was 19\%. Even with practice in the study (which might be expected to simulate specialist reporting) the incidence of extramural venous invasion seen when examining the new H\&E sections was only $24 \%$, but this increased significantly to $43 \%$ when H\&E/ elastica stained sections were examined (table 1). This implies that the use of an elastica stain is more crucial to increasing the sensitivity of detection of venous invasion than having the specimen reported by a moderately experienced specialist.

There is controversy in the literature about the prognostic relevance of intramural venous invasion. ${ }^{18}$ The elastic stain was particularly helpful in demonstrating this phenomenon. Although intramural venous invasion was not required to be recorded in the original reports, it was seen in eight cases when H\&E sections alone were examined in our study and in 30 cases examined with the special stain. Intramural vessels are smaller, thinner, and have less smooth muscle than their extramural counterparts. They are also found in the midst of the tumour (fig 2), as opposed to being at the leading edge of it, so it is not surprising that their invasion by tumour was much more difficult to detect when using only an H\&E stain.

Several authors have previously endorsed elastic tissue stains for the detection of venous invasion. In one of the few studies where the analysis of elastic stained sections was compared with findings using adjacent H\&E sections, Inoue and colleagues ${ }^{9}$ demonstrated that a Verhoeff van Gieson elastic (EVG) tissue stain significantly increased the incidence of observed venous invasion. This was particularly obvious in patients who developed haematogenous metastases during follow up. In this subgroup, the incidence of vascular invasion detected by H\&E was $31 \%$ but was $81 \%$ by EVG. When analysing the results of the cases stained only by $\mathrm{H} \& \mathrm{E}$, the correlation between the finding of vascular invasion and the development of subsequent distant metastases was not significant. However, significance was revealed when the elastic stained section results were analysed. The observation of the increased sensitivity of elastica stains was later confirmed by Sternberg and colleagues. ${ }^{10}$ In their study of stage IV colorectal cancers they identified a 38.5\% increase in observed venous invasion in serial sections stained by Weigert's elastica compared with H\&E stained ones.

\section{"The use of an elastica stain is probably more crucial to increasing the sensitivity of detection of venous invasion than having the specimen reported by a moderately experienced specialist"}

One influential study of venous invasion in rectal carcinomas $^{2}$ has been widely quoted ${ }^{9-11}$ as showing that the addition of elastic stains was "rarely found to be helpful" in detecting venous invasion. In Talbot's study, venous invasion was found in $52 \%$ of 703 cases. Intramural venous invasion only was found in $16 \%$ and extramural invasion, with or without intramural invasion, in $36 \%$. The prevalence of these findings in this extremely large series of consecutively excised specimens bears comparison with any other study using elastic stains (including this one). The detailed methods used in the study were given in another report $\mathrm{t}^{12}$ and are worthy of further analysis. In this prospective series an average of 4.8 tumour blocks were taken for each case, with at least one block taken to show the greatest penetration of the tumour, and one taken tangentially at the periphery of the tumour to identify transverse sections of veins emerging from the tumour (a practice no longer recommended ${ }^{7}$ ). In cases that on initial examination were found to be negative for venous invasion, further blocks, up to a maximum of eight, were taken. Initially, only $\mathrm{H} \& \mathrm{E}$ stains were done. If venous invasion was doubtful at a particular site the relevant block was re-cut and an elastica stain done. ${ }^{2}$ The study states that this stain was "rarely helpful" because re-cutting the paraffin wax blocks yielded sections that differed from the originalsthat is, the vein in question did not appear on the special stain. $^{2}$ This was a painstaking prospective study done by internationally renowned specialist pathologists who maximised their opportunity for detection of vascular invasion by the examination of additional blocks. They did not use serial sections to allow direct comparison of the sensitivity of detection of vascular invasion in elastica stained sections with H\&E stained sections. Thus, the study was not designed

\begin{tabular}{|c|c|c|c|c|c|c|c|c|}
\hline \multirow[b]{3}{*}{$c_{5}$} & \multirow[b]{3}{*}{$\mathrm{N}$} & \multicolumn{7}{|c|}{ Study results } \\
\hline & & \multicolumn{2}{|c|}{ Intramural VI } & \multicolumn{2}{|c|}{ Extramural VI } & \multicolumn{2}{|c|}{ Overall VI } & \multirow{2}{*}{$\begin{array}{l}\text { Extramural in original } \\
\text { report } \\
\text { H\&E (\%) }\end{array}$} \\
\hline & & H\&E (\%) & Elastica (\%) & H\&E (\%) & Elastica (\%) & H\&E (\%) & Elastica (\%) & \\
\hline Dukes's A & 5 & - & - & - & - & & - & - \\
\hline Dukes's B & 27 & $0(0)$ & $12(44)^{* * *}$ & $4(15)$ & $9(33)$ * & $4(15)$ & $14(52) * * *$ & $1(4)$ \\
\hline Dukes's C & 30 & $5(17)$ & $12(40)$ ** & $6(20)$ & $13(43)$ ** & $9(30)$ & $19(63) * * *$ & $9(30)$ \\
\hline Dukes's D & 13 & $3(23)$ & $6(46)$ & $8(61)$ & 10 (77) & $8(62)$ & 10 (77) & $4(31)$ \\
\hline Total & 75 & $8(11)$ & $30(40) * * \star$ & $18(24)$ & $32(43) * * *$ & $21(27)$ & $43(57) * * *$ & 14 (19) \\
\hline
\end{tabular}


Table 3 Venous invasion (VI) on elastica stained sections related to number of blocks examined

\begin{tabular}{lll}
\hline No of blocks & No of cases & No with VI (\%) \\
\hline $2-3$ & 28 & $14(50)$ \\
$4-5$ & 31 & $20(65)$ \\
$6-11$ & 16 & $9(56)$ \\
Total & 75 & $43(57)$ \\
\hline
\end{tabular}

to test whether there might be additional sensitivity with the use of elastica stains, and those authors who have quoted it out of context as showing that elastica stains were rarely helpful have misrepresented both the design and conclusions of the paper.

Petersen et al have shown that both intramural and extramural venous invasion influence the prognostic index in Dukes's B colon carcinomas, which correlates with survival. If this finding is confirmed then patients with a high prognostic index (and therefore worse prognosis) may be considered for adjuvant chemotherapy. In this setting, the ability of pathologists to identify venous invasion will have a direct influence on patient care.

In our present study, examination of only H\&E sections demonstrated vascular invasion in four of 27 patients with Dukes's B tumours. By comparison, 14 of the 27 tumours were shown to have vascular invasion when elastica stains were incorporated $(\mathrm{p}<0.001)$. In Petersen's study, vascular invasion was detected, without the use of special stains, in $43 \%$ of cases. The colons of all 268 cases in their study were received by one highly respected, specialist pathologist who selected all the blocks and reported the histology. Our study suggests that only the use of special stains will allow the more general pathologist to achieve similar sensitivity in the detection of venous invasion (and thus an accurate assessment of the prognostic index) in the routine reporting situation.

The present findings imply that serial sections of tumour blocks in colorectal carcinoma should be stained routinely by $\mathrm{H} \& \mathrm{E}$ and an elastic stain to ensure an acceptable level of sensitivity in the detection of venous invasion. Arguably, this recommendation should be incorporated into future guidelines for the histopathological reporting of these specimens.
Take home messages

- The use of haematoxylin and eosin (H\&E) and elastica stained serial sections increased the detection of venous invasion in colorectal carcinomas compared with H\&E only

- Arguably, this recommendation should be incorporated into future guidelines for the histopathological reporting of these specimens

\section{Authors' affiliations}

D G Vass, R Ainsworth, D Murray, A K Foulis, Department of Pathology, Royal Infirmary, Glasgow G4 OSF, UK

J H Anderson, Department of Surgery, Royal Infirmary, Glasgow

Correspondence to: Dr A K Foulis, Department of Pathology, Royal Infirmary, Glasgow G4 OSF, UK; alan.foulis@northglasgow.scot.nhs.uk

Accepted for publication 29 January 2004

\section{REFERENCES}

1 Brown CF, Warren S. Visceral metastasis from rectal carcinoma. Surg Gynaecol Obstet 1938;66:611-21.

2 Talbot IC, Ritchie S, Leighton MH, et al. The clinical significance of invasion of veins by rectal cancer. Br J Surg 1980;67:439-42.

3 Krasna MJ, Flanbaum L, Cody RP, et al. Vascular and neural invasion in colorectal carcinoma. Cancer 1988;61:1018-23.

4 Shirouzu K, Isomoto $\mathrm{H}$, Kakegawa $\mathrm{T}$, et al. A prospective clinicopathological study of venous invasion in colorectal cancer. Am J Surg 1991;162:216-22.

5 Horn A, Dahl O, Morild I. Venous and neural invasion as predictors of recurrence in rectal adenocarcinoma. Dis Colon Rectum 1991;34:798-804.

6 Ouchi K, Sugawara T, Ono H, et al. Histologic features and clinical significance of venous invasion in colorectal carcinoma with hepatic metastasis. Cancer 1996;78:2313-17.

7 Quirke P, William GT. Minimum data set for colorectal cancer. London: The Royal College of Pathologists, 2000.

8 Petersen VC, Baxter KJ, Love SB, et al. Identification of objective pathological prognostic determinants and models of prognosis in Dukes' B colon cancer. Gut 2002;51:65-9

9 Inove T, Mori M, Shimono R, et al. Vascular invasion of colorectal carcinoma readily visible with certain stains. Dis Colon Rectum 1992;35:34-9.

10 Sternberg A, Amar M, Alfici R, et al. Conclusions from a study of venous invasion in stage IV colorectal adenocarcinoma. J Clin Pathol 2002;55:17-21.

11 Minsky B, Mies C. The clinical significance of vascular invasion in colorectal cancer. Dis Colon Rectum 1989;32:7994-803.

12 Talbot IC, Ritchie S, Leighton M, et al. Invasion of veins by carcinoma of rectum: method of detection, histological features and significance. Histopathology 1981;5:141-63. 